\title{
Clinical Significance of Ubiquitin-associated Protein 2-like in Patients With Uterine Cervical Cancer
}

\author{
KOSUKE YOSHIDA $^{1}$, HIROAKI KAJIYAMA ${ }^{1}$, ERI INAMI ${ }^{2}$, SATOSHI TAMAUCHI ${ }^{1}$, YOSHIKI IKEDA ${ }^{1}$, \\ NOBUHISA YOSHIKAWA ${ }^{1}$, KIMIHIRO NISHINO ${ }^{1}$, FUMI UTSUMI $^{3}$, KAORU NIIMI $^{1}$, SHIRO SUZUKI ${ }^{1}$, \\ KIYOSUMI SHIBATA ${ }^{3}$, AKIHIRO NAWA ${ }^{2}$ and FUMITAKA KIKKAWA ${ }^{1}$ \\ ${ }^{1}$ Department of Obstetrics and Gynecology, Nagoya University Graduate School of Medicine, Nagoya, Japan; \\ ${ }^{2}$ Bell Research Center for Reproductive Health and Cancer, Nagoya, Japan; \\ ${ }^{3}$ Department of Obstetrics and Gynecology, Fujita Health University Bantane Hospital, Nagoya, Japan
}

\begin{abstract}
Background: Ubiquitin-associated protein 2-like (UBAP2L) has been demonstrated to be associated with the progression of multiple types of cancer. However, the function of UBAP2L in uterine cervical cancer remains unclear. Materials and Methods: Between 2005 and 2015, 84 patients who underwent surgery were included in this study. The patients were stratified into two groups on the basis of immunohistochemical staining for UBAP2L, and survival analysis was performed. Moreover, loss-of-function analysis was performed using the cervical cancer cell lines CaSki and SiHa. Results: Based on immunohistochemistry, the overall survival in patients with low UBAP2L expression was significantly longer than that of those with high UBAP2L expression ( $p=0.045)$. The in vitro experiment revealed that knockdown of $U B A P 2 L$ remarkably inhibited cell proliferation in both live cell imaging and the MTS assay. Conclusion: Patients with high UBAP2L expression had unfavorable prognosis and UBAP2L appears to play an important role in proliferation.
\end{abstract}

Cervical cancer is a common malignancy observed among females worldwide. The mortality rates associated with uterine cervical cancer have declined due to the widespread use of cancer screening for the prevention and early detection of cervical cancer (1). However, approximately one-third of patients experience recurrence within 5 years,

This article is freely accessible online.

Correspondence to: Hiroaki Kajiyama, MD, Ph.D., Department of Obstetrics and Gynecology, Nagoya University Graduate School of Medicine, Tsuruma-cho 65, Showa-ku, Nagoya 466-8550, Japan. E-mail:kajiyama@med.nagoya-u.ac.jp

Key Words: Cervical cancer, ubiquitin-associated protein 2-like, prognostic factor, RNA interference, proliferation. and their post-recurrence oncological outcome is generally poor (2-4). Therefore, the oncological outcome for these patients is far from satisfactory.

Ubiquitin-associated protein 2-like (UBAP2L), also known as NICE-4, is a widely conserved protein with a ubiquitin-associated domain and multiple RGG/RG repeats at the $N$-terminus. UBAP2L has also been shown to aggregate after proteasome inhibition (5). The ubiquitinproteasome pathway is commonly used for protein degradation in eukaryotes and is considered to be involved in multiple cellular processes (6-8). In addition, recent studies have confirmed that $U B A P 2 L$ functions as an oncogene and is associated with various types of cancer, including prostate cancer (9), glioma (10), hepatocellular carcinoma $(11,12)$, lung adenocarcinoma (13), and breast carcinoma (14). Therefore, inhibition of the ubiquitinproteasome system is a promising anticancer strategy, and several proteasome inhibitors have been approved for clinicaI use (15-17). In uterine cervical cancer, oncogenesis by human papillomavirus (HPV) is mediated by ubiquitin ligase pathways alteration (6). However, the function and clinical impact of UBAP2L in uterine cervical cancer remains unknown.

In this study, we investigated UBAP2L expression in surgical specimens of cervical cancer and its effect on survival. Subsequently, we performed loss-of-function analysis and investigated the effect of $U B A P 2 L$ knockdown on cell proliferation and cell-cycle arrest.

\section{Materials and Methods}

Patient selection and immunohistochemistry $(I H C)$. We retrospectively reviewed all the records of patients with cervical cancer who were initially treated in our hospital from 2005 to 2015. This study was approved by the Ethics Committee of our Institute (approval no.: 2017-0053), and approval for an opt-out consent method was provided. Informed consent was obtained by the opt-out consent method on Nagoya University website. We included 84 patients who 
underwent radical hysterectomy ( $\mathrm{RH})$, and for whom histological examination revealed pT1b1 to pT2b squamous cell carcinoma (SCC). Patients who had received neoadjuvant therapy and had no residual tumor in the RH specimen after conization were excluded.

Archival formalin-fixed, paraffin-embedded tumor tissue obtained at RH was used in this study, and sections of $4-\mu \mathrm{m}$ thickness were prepared using a microtome. The sections were deparaffinized, rehydrated, subjected to antigen retrieval in $10 \mathrm{mM}$ sodium citrate $\left(\mathrm{pH} \mathrm{6.0)}\right.$ for $20 \mathrm{~min}$ at $95^{\circ} \mathrm{C}$ in a microwave, and treated with $0.3 \%$ hydrogen peroxide in methanol for 20 min after being washed with phosphate-buffered saline (PBS). The sections were then blocked with goat serum using Histofine $\mathrm{SAB}-\mathrm{PO}(\mathrm{R})$ kit according to the manufacturer protocol (Nichirei, Tokyo, Japan) and incubated with a polyclonal rabbit antibody to UBAP2L (HPA035068; Sigma-Aldrich, St. Louis, MO, USA) diluted by PBS at $4{ }^{\circ} \mathrm{C}$ overnight. After rinsing with PBS, the sections were incubated with a secondary antibody, and then peroxidase-labeled streptavidin. The sections were then rinsed with PBS and developed by the 3,3'-diaminobenzidine (DAB) substrate-chromogen (Nichirei, Tokyo, Japan). After rinsing in water, the sections were incubated with hematoxylin, dehydrated, and mounted. Based on the IHC activity, a four-tiered semi-quantitative score was assigned according to the intensity and area of stained cells, as follows: The staining intensity was scored as: 0 , negative; 1 , weak; 2 , medium; or 3 , strong. The percentage of the staining area was scored as 'focal' (1$10 \%)$, 'sporadic' $(11-50 \%)$, and diffuse $(>51 \%)$ relative to the total tumor area (Figure 1A). All photographs were taken using Zeiss Axio Imager.A1 (Carl Zeiss, Tokyo, Japan).

The Cancer Genome Atlas (TCGA) database. Normalized RNA-Seq expression data and clinical survival information of tumor samples were downloaded from cBioPortal (http://www.cbioportal.org). Using data of 187 patients with stage I or II SCC, we performed survival analysis stratified by $U B A P 2 L$ mRNA expression (low: zscore $<0$ or high: $\mathrm{z}$-score $\geq 0$ ).

Cell culture and small interfering RNA (siRNA) transfection. Cervical cancer cell lines, CaSki and SiHa were purchased from the American Type Culture Collection (Manassas, VA, USA). These cell lines were maintained in RPMI (Sigma-Aldrich) containing 10\% fetal bovine serum (Sigma-Aldrich) and penicillin-streptomycin solution (Meiji Seika Pharma, Tokyo, Japan). These cell lines were confirmed to be negative for mycoplasma contamination.

To suppress $U B A P 2 L$, two different Silencer Select Pre-designed siRNAs for UBAP2L were used (siRNA IDs were s19176 and s230223; Thermo Fisher Scientific, Waltham, MA, USA). The sequences of s19176 and s230223 were 5"-GCAGCUUCCUUACUGACGA-3' (siUBAP2L-1) and 5'-GCAGAAUACCCUUUCAUCA-3' (siUBAP2L2), respectively. Silencer Negative Control No. 1 siRNA (Thermo Fisher Scientific) was used as a control (siCtrl). Cells were transfected with $3 \mathrm{nM}$ siRNA using Lipofectamin RNAi Max (Thermo Fisher Scientific, Waltham, MA). Cells were used for further analysis $72 \mathrm{~h}$ after transfection.

Western blotting. Total protein was extracted from cell lines using RIPA Protein Lysis solution (Thermo Fisher Scientific, Waltham, MA). Protein quantification was carried out using BCA protein assay kit (Wako, Osaka, Japan). After quantification, $30 \mu \mathrm{g}$ of total proteins were separated in $10 \%$ sodium dodecyl sulphate-polyacrylamide gel electrophoresis gel electrophoresis and transferred onto PVDF membranes. The transferred polyvinylidene fluoride membranes were treated with $5 \%$ skim milk for $30 \mathrm{~min}$ at room temperature before being incubated overnight at $4^{\circ} \mathrm{C}$ with a rabbit anti-UBAP2L, purified in a previous study (18).

The membranes were washed thrice with phosphate-buffered saline with Tween 20 and then incubated with horseradish peroxidaseconjugated goat anti-rabbit IgG (Cell Signaling Technology, Danvers, MA, USA) for $1 \mathrm{~h}$ at room temperature. Finally, the immunoreactive bands were detected using enhanced chemiluminescence.

Proliferation assay. Transfected cells were reseeded in a 96-well plate at a density of 1,000 cells/well and incubated overnight. The plate was moved into an IncuCyte ZOOM (Essen BioScience, Ann Arbor, MI, USA) for live cell imaging, and images of each well were recorded every hour for a total of $72 \mathrm{~h}$. Finally, the confluency of each well was determined using IncuCyte ZOOM 2018 software.

For the MTS assay, $10 \mu \mathrm{l}$ of $5 \mathrm{mg} / \mathrm{ml}$ CellTiter 96 Aqueous One Solution (Promega, Madison, WI, USA) was added to each well and incubated with cells for $2 \mathrm{~h}$. The optical density (OD) of each well was determined using a spectrophotometer at a wavelength of $490 \mathrm{~nm}$.

Cell-cycle analysis. Transfected cells were reseeded in 6-cm dish and grown for 24 consecutive $h$. The cells were harvested following trypsinization, washed with $1 \%$ bovine serum albumin (BSA; Wako, Osaka, Japan)/PBS, and fixed in cold $70 \%$ ethanol at $4{ }^{\circ} \mathrm{C}$ overnight. The fixed cells were washed with $1 \%$ BSA/PBS and collected by centrifugation $(300 \times g$ for $30 \mathrm{~min})$. Cells were resuspended in propidium iodide and RNase solution (BD Biosciences, San Jose, CA, USA) and used for FACS Canto2 flow cytometer analysis (BD Biosciences). The obtained data were analyzed with the ModFit LT DNA analysis program (Verity Software House, Topsham, ME, USA).

Statistical analysis. Statistical analysis was performed with SPSS version 25 (IBM, Armonk, NY, USA). The baseline differences between patients stratified by UBAP2L expression were assessed by Student's $t$-tests and Mann-Whitney U-tests. Kaplan-Meier curves and log-rank test were used for the survival analysis. Three parameters of survival were studied: overall survival (OS), progression-free survival (PFS), and disease-free survival (DFS). OS was defined as the time from initial surgery to death from any cause and PFS was the time from initial surgery to tumor progression or death from any cause. In the analysis of the TCGA database, OS and DFS data were obtained from the database. For the in vitro assay, differences were assessed by Student's $t$-tests. A value of $p<0.05$ was taken as statistically significant.

\section{Results}

A total of 82 patients with SCC of the uterine cervix were enrolled. All the patients underwent RH without neoadjuvant therapy, and their surgical specimens were used for IHC. UBAP2L expression was strong in $21(25.0 \%)$, moderate in $24(28.6 \%)$, weak in $29(34.5 \%)$, and negative in $10(11.9 \%)$ patients. Representative IHC images are shown in Figure 1A. The association between UBAP2L expression and survival was also evaluated by stratifying patients into groups with low UBAP2L (negative and weak) and those with high UBAP2L (moderate and strong). 
A
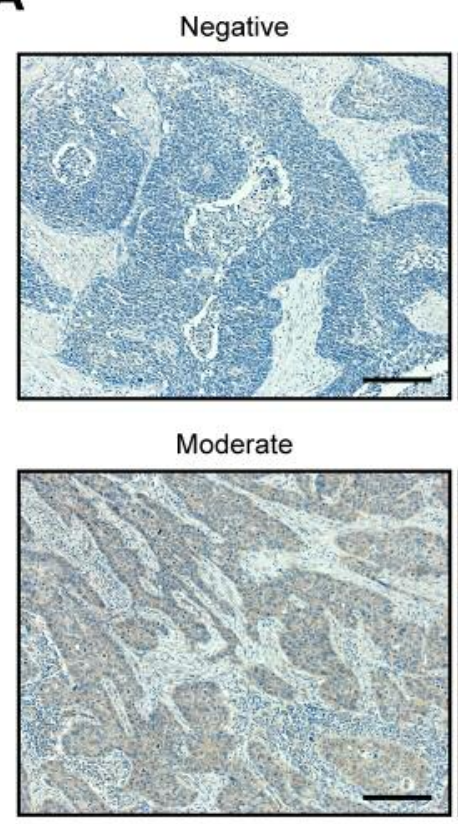

Weak
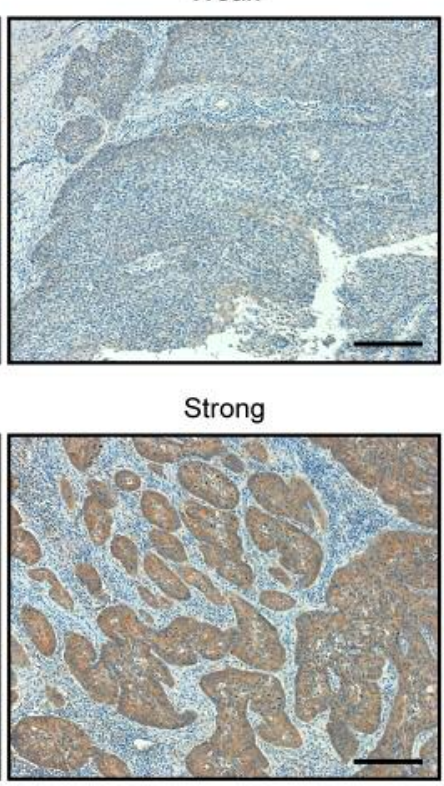

Intensity

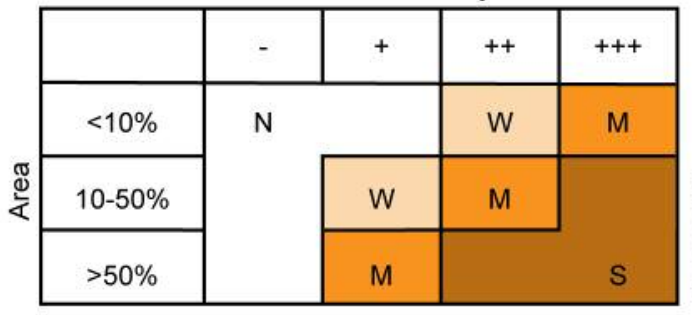

N, Negative

W, Weak

M, Moderate

S, Strong
B

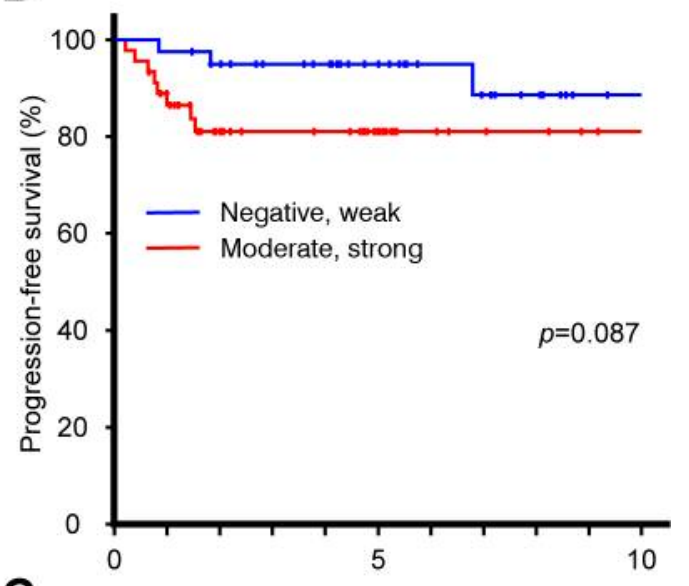

C

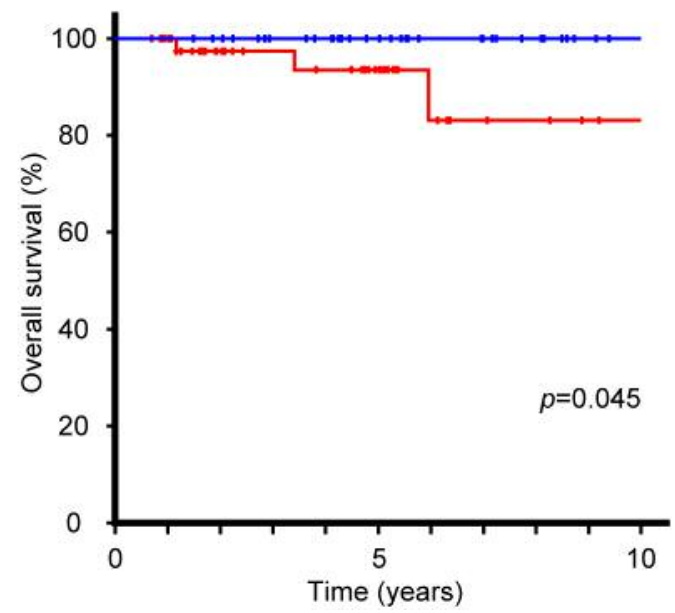

Figure 1. Ubiquitin-associated protein 2-like (UBAP2L) expression in patients with cervical cancer and survival impact. A: Representative images of immunohistochemistry and evaluation of immunohistochemistry (Scale bars=200 $\mu \mathrm{m}$ ). Kaplan-Meier curves showing progression-free (B) and overall $(C)$ survival stratified by UBAP2L expression.

In the 59.1-month median follow-up period (range=8.2153.3 months), 11 patients experienced recurrence and three patients died. Patient characteristics, including age, pT, tumor size, $\mathrm{pN}$, lymphovascular space invasion, and treatment, were not significantly different between the low and high UBAP2L groups (Table I). Kaplan-Meier curves indicated that PFS in the low UBAP2L group tended to be longer than that of the high UBAP2L group $(p=0.087)$ (Figure 1B). All three patients who died were in the high UBAP2L group, and Kaplan-Meier curves revealed that the difference in OS between the two groups was significant $(p=0.045)$ (Figure 1C).

To validate our result from IHC, we performed survival analysis using the TCGA database. According to the database, 53 patients had low $U B A P 2 L$ mRNA expression (zscore $<0)$ and 134 patient high expression $(\mathrm{z}$-score $\geq 0$ ).
Kaplan-Meier curves demonstrated significantly longer DFS and OS in the low expression than the high expression group ( $p=0.014$ and $p=0.031$, respectively) (Figure 2 ).

We next evaluated the role of UBAP2L expression in vitro. The efficacy of $U B A P 2 L$ down-regulation was confirmed by western blot (Figure 3A). Representative images of live cell imaging are shown in Figure 3B, and the confluency for each image was calculated. At $72 \mathrm{~h}$, the siCtrl CaSki cells had proliferated 5.69 times, while the siUBAP2L-1 and -2 CaSki cells had proliferated 2.76 and 3.60 times, respectively. Therefore, knockdown of $U B A P 2 L$ significantly inhibited the proliferation of CaSki cells $(p<0.001$ for both groups, Figure 3C). Similar growth inhibition was observed in the SiHa cells. siCtrl, siUBAP2L1, and -2 SiHa cells had proliferated 6.03, 4.92, and 3.58 times, respectively (siUBAP2L-1 and -2 : $p=0.002$ and 
Table I. Baseline characteristics of patients with cervical cancer.

\begin{tabular}{|c|c|c|c|c|}
\hline & \multicolumn{4}{|c|}{ UBAP2L } \\
\hline & & $\begin{array}{c}\text { Negative, weak } \\
\quad(n=39)\end{array}$ & $\begin{array}{c}\text { Moderate, strong } \\
\quad(\mathrm{n}=45)\end{array}$ & $p$-Value \\
\hline Age, years & Median (range) & $50(21-68)$ & $45(20-73)$ & 0.631 \\
\hline \multirow{2}{*}{$\mathrm{pT}, \mathrm{n}(\%)$} & $1 b$ & $21(53.8 \%)$ & $24(53.3 \%)$ & 0.963 \\
\hline & $2 a, 2 b$ & $18(46.2 \%)$ & $21(46.7 \%)$ & 0.844 \\
\hline \multirow[t]{2}{*}{ Tumor size } & $<4 \mathrm{~cm}$ & $36(92.3 \%)$ & $41(91.1 \%)$ & 0.356 \\
\hline & $\geq 4 \mathrm{~cm}$ & $3(7.7 \%)$ & $4(8.9 \%)$ & 0.161 \\
\hline \multirow[t]{2}{*}{$\mathrm{pN}, \mathrm{n}(\%)$} & 0 & $28(71.8 \%)$ & $28(62.2 \%)$ & 0.512 \\
\hline & 1 & $11(28.2 \%)$ & $17(37.8 \%)$ & 0.631 \\
\hline \multirow[t]{2}{*}{ LVSI, n (\%) } & - & $11(28.2 \%)$ & $7(15.6 \%)$ & 0.963 \\
\hline & + & $28(71.8 \%)$ & $38(84.4 \%)$ & 0.844 \\
\hline \multirow[t]{2}{*}{ Treatment, n (\%) } & $\mathrm{RH}$ & $8(20.5 \%)$ & $12(26.7 \%)$ & 0.356 \\
\hline & $\mathrm{RH}+\mathrm{CCRT}$ & $31(79.5 \%)$ & $33(73.3 \%)$ & 0.161 \\
\hline
\end{tabular}

LVSI: Lymphovascular space invasion; RH: radical hysterectomy; CCRT: concurrent chemoradiotherapy.

A

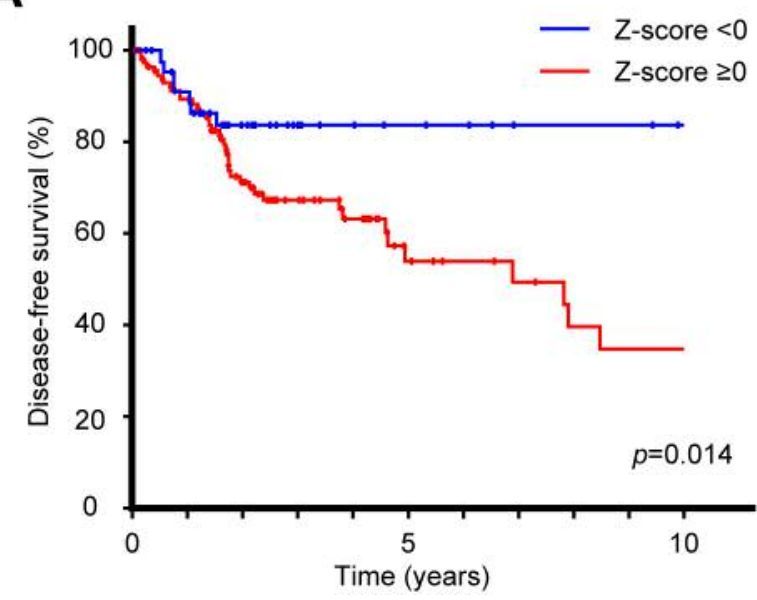

B

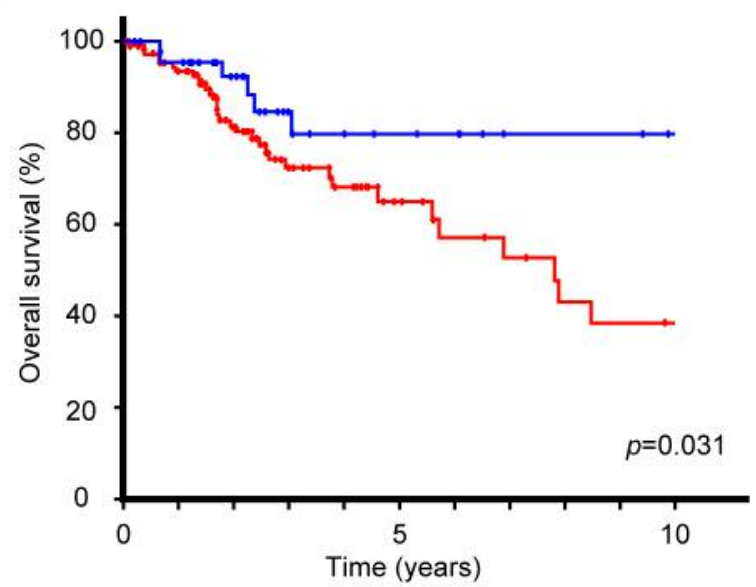

Figure 2. Survival impact of ubiquitin-associated protein 2-like (UBAP2L) mRNA expression from the The Cancer Genome Atlas (TCGA) database. Kaplan-Meier curves showing disease-free $(A)$ and overall $(B)$ survival stratified by UBAP2L mRNA expression Z-score.

$p<0.001$, respectively, Figure 3C). The results of the MTS assay also supported this growth-inhibitory effect. At $72 \mathrm{~h}$, compared with siCtrl CaSki, the OD490 values of siUBAP2L-1 and -2 cells decreased to $65.2 \%$ and $66.8 \%$, respectively $(p<0.001$ for both groups, Figure 3D). Moreover, compared with siCtrl SiHa cells, the OD490 values of siUBAP2L-1 and -2 cells decreased to $78.7 \%$ and $54.5 \%$, respectively ( $p<0.001$ in both groups, Figure 3D). Therefore, knockdown of $U B A P 2 L$ significantly inhibited cell proliferation in both cell lines.

Finally, in order to elucidate the potential mechanism underlying growth inhibition, the effects of $U B A P 2 L$ knockdown on the cell-cycle distribution were evaluated. In both cell lines, the cell population in the $\mathrm{G}_{0} / \mathrm{G}_{1}$ phase was significantly reduced (CaSki siUBAP2L-1 and $-2: p=0.006$ and $p=0.026$, respectively, and SiHa siUBAP2L-1 and -2 : $p=0.004$ and $p=0.007$, respectively, Figure 4). Moreover, the cell population in the $S$ phase was significantly increased in siUBAP2L CaSki cells ( $p=0.002)$, and those in $\mathrm{G}_{2} / \mathrm{M}$ phase tended to increase in both cell lines (Figure 4).

\section{Discussion}

Previous studies demonstrated that UBAP2L is involved in the ubiquitin-proteasome system that degrades most intracellular proteins $(5,19)$. However, the function of UBAP2L in cervical 
A

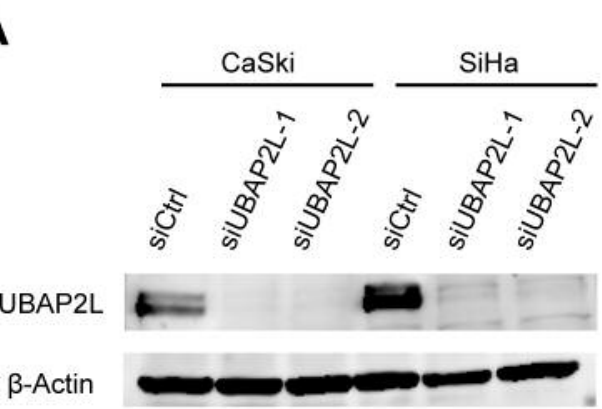

B
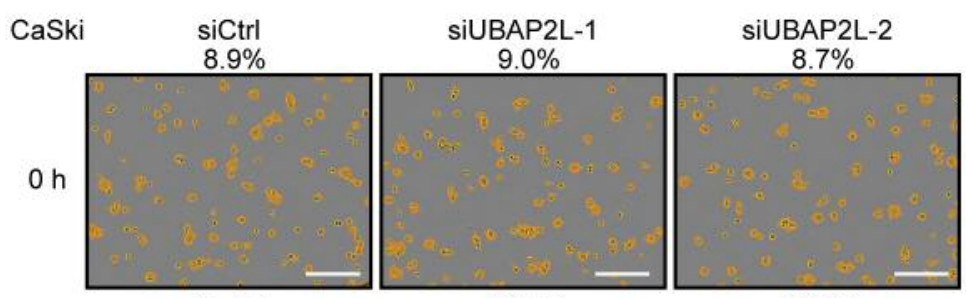

B-Actin

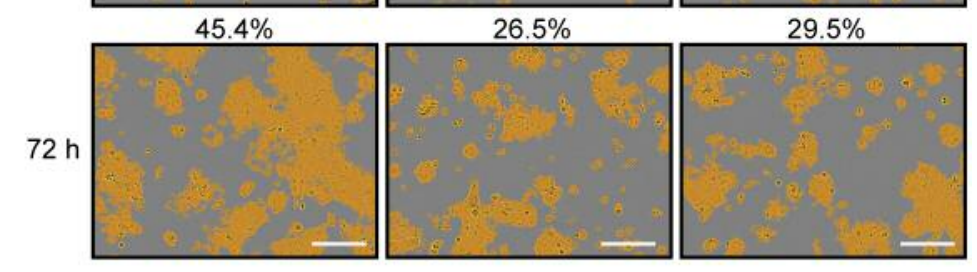

C
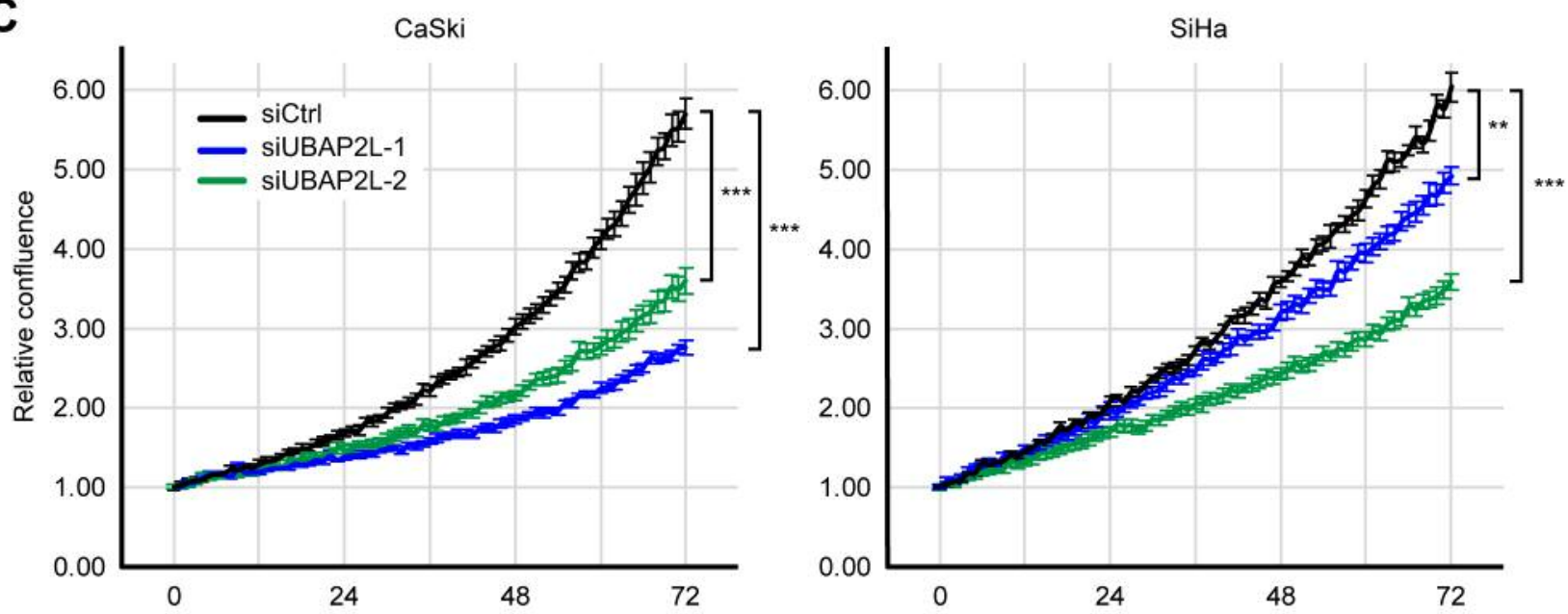

D
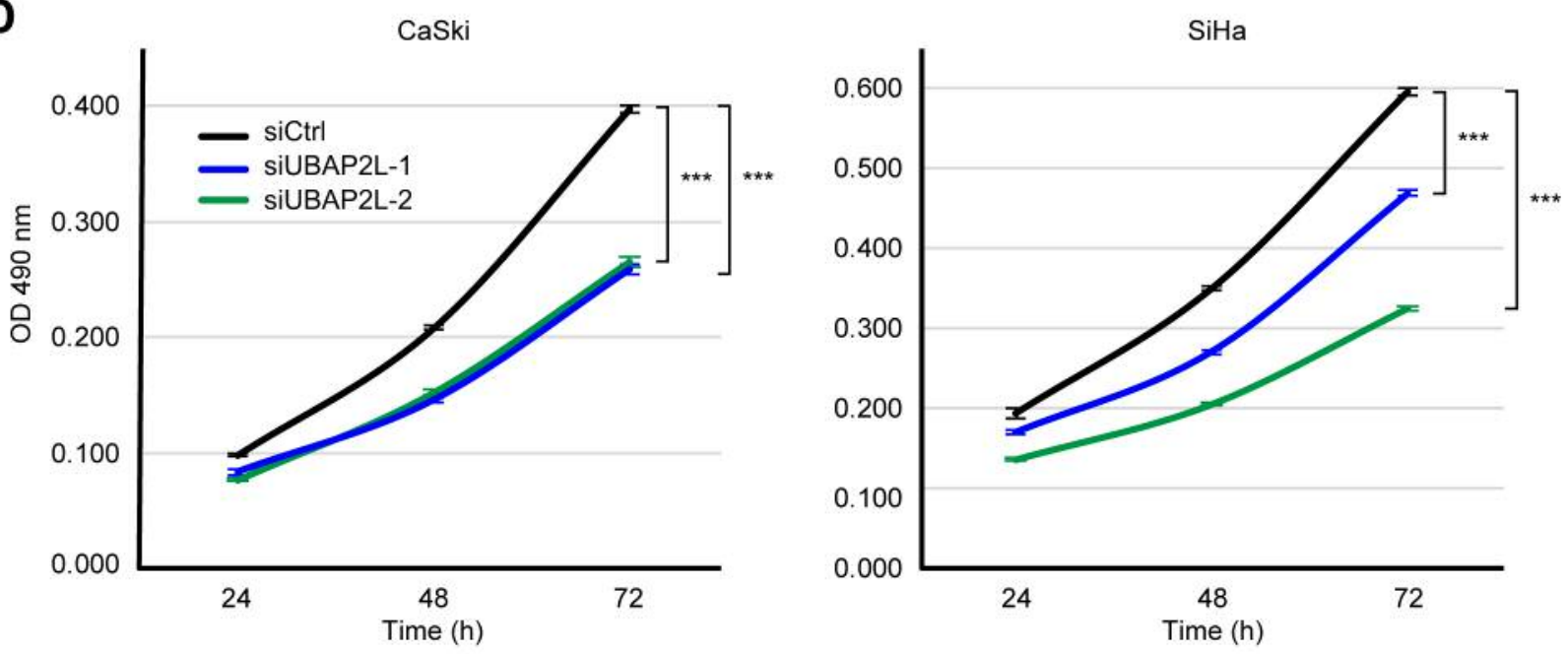

Figure 3. Down-regulation of ubiquitin-associated protein 2-like (UBAP2L) using small interfering RNA (siRNA) inhibited the proliferation of cervical cancer cells. A: Western blot analysis of the UBAP2L protein level in the cervical cancer cell lines CaSki and SiHa. Beta actin was used as a loading control. B: Representative images of CaSki cells analyzed by IncuCyte ZOOM. The confluence values are shown (scale bars, 300 um). $C$ : Growth curves based on the relative confluence generated by the IncuCyte ZOOM. D: Growth curves based on MTS assay. Data represent the mean \pm standard error. Significantly different at $* * p<0.01, * * * p<0.001$ vs. siCtrl. 


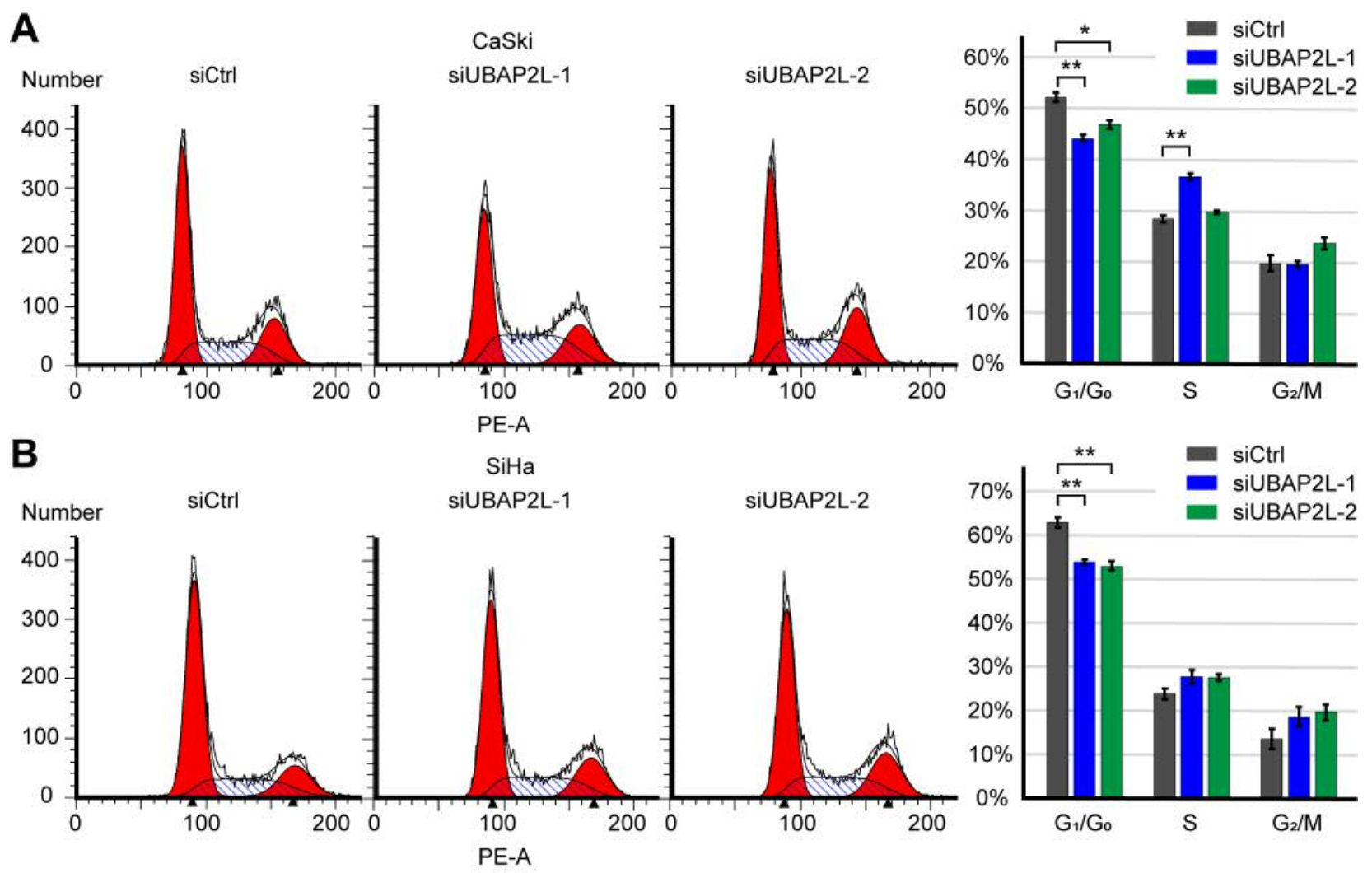

Figure 4. Down-regulation of ubiquitin-associated protein 2-like (UBAP2L) induced cell-cycle arrest. The cell-cycle distribution of CaSki (A) and $\mathrm{SiHa}(B)$ cells was analyzed by flow cytometry. Data represent the mean \pm standard error. Significantly different at ${ }^{*} p<0.05,{ }^{* *} p<0.01 \mathrm{vs}$. siCtr.

cancer has not yet been elucidated. To the best of our knowledge, the present study is the first to evaluate the impact of UBAP2L expression in cervical cancer.

Initially, IHC revealed that high expression of UBAP2L was associated with significantly poor prognosis in patients with cervical cancer. However, there were only a few patients who experienced recurrence or death in our cohort. Thus, we used data from the TCGA database to validate the poor prognosis associated with high UBAP2L expression. According to previous reports, $U B A P 2 L$ is up-regulated in hepatocellular carcinoma compared with normal tissues, and the prognosis of patients with high UBAP2L expression is significantly poorer than those with low expression $(11,12)$. Moreover, a similar result was also reported in patients with lung adenocarcinoma (13). Therefore, overexpression of UBAP2L is considered to be associated with poor prognosis and to contribute to cancer progression.

In order to elucidate the function of UBAP2L in cervical cancer, loss-of-function analysis was performed in vitro. The results demonstrated that down-regulation of $U B A P 2 L$ reduced proliferation and led to cell-cycle arrest in cervical cancer cell lines. These results were consistent with previous reports which used several cell lines $(9,10,14)$. It has been shown that the arginine methylation of UBAP2L by protein arginine $N$-methyltransferase 1 is required for the proper distribution of chromosomes during mitosis (18). Hence, UBAP2L is considered to play an important role in mitosis; thus, the finding that down-regulation of $U B A P 2 L$ resulted in $\mathrm{G}_{2} / \mathrm{M}$ arrest was typical $(9,14)$. However, several cell lines have been shown to undergo $G_{0} / G_{1}$ or $S$ phase arrest as a result of $U B A P 2 L$ down-regulation $(9,10)$. Therefore, UBAP2L function might differ depending on the cell type, and UBAP2L might have additional unknown functions. In addition, differently from other malignancies in the previous reports (9-14), cervical cancer is caused by HPV, and its oncogene $E 6$ and $E 7$ target key cell-cycle control proteins (6, 20). Therefore, further evaluation of the association between UBAP2L and HPV oncogenes would be interesting.

Recently, a report indicated that UBAP2L modulates stress granule (SG) assembly (21). SGs are non-membranous cytoplasmic foci composed of non-translating messenger ribonucleoproteins (22). Various environmental stresses, such as heat shock, oxidative stress, and chemotherapeutic drugs, induce SG assembly to minimize stress-related damage and 
promote cell survival, but X-ray irradiation does not (22-24). However, SG formation occurs under hypoxic conditions, and SG depolymerization, caused by radiation-induced reoxygenation, is involved in hypoxia inducible factor-1 (HIF1) activation (23-25). As a result, HIF1-regulated cytokines enhance radioresistance (25); hence UBAP2L might be associated with both chemo- and radioresistance. Therefore, not only increased proliferation but also treatment resistance due to UBAP2L might be associated with poor prognosis of patients with high UBAP2L expression, although we did not evaluate SG and UBAP2L-related treatment resistance in this study.

The present study has several limitations. Firstly, in IHC analysis, we only evaluated patients with early-stage disease because we used surgical specimens. Thus, the nature of UBAP2L expression in advanced or recurrent cervical cancer remains unknown. Secondly, as described previously, because there were only a few patients who experienced recurrence or death, the statistical power was relatively weak. Thirdly, in the TCGA database, the details of the patients are unknown, and potential bias might have existed. Therefore, further studies examining the use of UBAP2L as a prognostic factor are desired. In the in vitro analysis, we evaluated the impact of UBAP2L on cell proliferation and the cell cycle. Additional functions of UBAP2L, such as in SGs and epithelial-mesenchymal transition, should be evaluated in future studies.

In conclusion, we demonstrated that UBAP2L plays a critical role for proliferation in cervical cancer, and as a result, UBAP2L expression was correlated with poor prognosis. UBAP2L might be a potential therapeutic target, and further studies are desired.

\section{Conflicts of Interest}

None.

\section{Authors' Contributions}

KY contributed to acquisition of data and was a major contributor in writing the article. HK contributed to conception and design and gave final approval of the version to be published. EI contributed to acquisition of data. ST involved in revising the article critically. YI contributed analysis and interpretation of data. NY contributed to acquisition of data. $\mathrm{KN}$ contributed analysis and interpretation of data. FU contributed to conception and design and involved in revising the article critically. KN, SS, KS, and AN contributed analysis and interpretation of data. FK gave final approval of the version to be published. All Authors read and approved the final article.

\section{Acknowledgements}

The Authors thank Enago (https://www.enago.jp/) for English editing of this article.

\section{References}

1 Siegel RL, Miller KD and Jemal A: Cancer statistics, 2016. CA Cancer J Clin 66(1): 7-30, 2016. PMID: 26742998. DOI: $10.3322 /$ caac. 21332

2 Benedet JL, Odicino F, Maisonneuve P, Beller U, Creasman WT, Heintz AP, Ngan HY and Pecorelli S: Carcinoma of the cervix uteri. Int J Gynaecol Obstet 83 Suppl 1: 41-78, 2003. PMID: 14763169. DOI: 10.1016/s0020-7292(03)90115-9

3 Waggoner SE: Cervical cancer. Lancet 361(9376): 2217-2225, 2003. PMID: 12842378. DOI: 10.1016/s0140-6736(03)13778-6

4 Yoshida K, Kajiyama H, Utsumi F, Niimi K, Sakata J, Suzuki S, Shibata K and Kikkawa F: A post-recurrence survival-predicting indicator for cervical cancer from the analysis of 165 patients who developed recurrence. Mol Clin Oncol 8(2): 281-285, 2018. PMID: 29435288. DOI: $10.3892 / \mathrm{mco} .2017 .1530$

5 Wilde IB, Brack M, Winget JM and Mayor T: Proteomic characterization of aggregating proteins after the inhibition of the ubiquitin proteasome system. J Proteome Res 10(3): 10621072, 2011. PMID: 21204586. DOI: 10.1021/pr1008543

6 Mani A and Gelmann EP: The ubiquitin-proteasome pathway and its role in cancer. J Clin Oncol 23(21): 4776-4789, 2005. PMID: 16034054. DOI: 10.1200/jco.2005.05.081

7 Rodriguez-Gonzalez A, Cyrus K, Salcius M, Kim K, Crews CM, Deshaies RJ and Sakamoto KM: Targeting steroid hormone receptors for ubiquitination and degradation in breast and prostate cancer. Oncogene 27(57): 7201-7211, 2008. PMID: 18794799. DOI: $10.1038 /$ onc.2008.320

8 Lin GL, Wang H, Dai J, Li X, Guan M, Ding Q, Wang HX and Fang H: Upregulation of Ubap $2 l$ in bone marrow mesenchymal stem cells promotes functional recovery in rats with spinal cord injury. Curr Med Sci 38(6): 1081-1089, 2018. PMID: 30536073. DOI: $10.1007 / \mathrm{s} 11596-018-1987-\mathrm{x}$

9 Li D and Huang Y: Knockdown of ubiquitin associated protein 2-like inhibits the growth and migration of prostate cancer cells. Oncol Rep 32(4): 1578-1584, 2014. PMID: 25069639. DOI: 10.3892/or.2014.3360

10 Zhao B, Zong G, Xie Y, Li J, Wang $\mathrm{H}$ and Bian E: Downregulation of ubiquitin-associated protein 2-like with a short hairpin RNA inhibits human glioma cell growth in vitro. Int J Mol Med 36(4): 1012-1018, 2015. PMID: 26310274. DOI: 10.3892/ijmm.2015.2323

11 Wang W, Zhang M, Peng $\mathrm{Y}$ and He J: Ubiquitin associated protein 2-like (ubap2l) overexpression in patients with hepatocellular carcinoma and its clinical significance. Med Sci Monit 23: 4779-4788, 2017. PMID: 28981479. DOI: 10.12659/ msm.907071

12 Ye T, Xu J, Du L, Mo W, Liang Y and Xia J: Downregulation of $U B A P 2 L$ inhibits the epithelial-mesenchymal transition via SNAIL1 regulation in hepatocellular carcinoma cells. Cell Physiol Biochem 41(4): 1584-1595, 2017. PMID: 28334716. DOI: $10.1159 / 000470824$

13 Aucagne R, Girard S, Mayotte N, Lehnertz B, Lopes-Paciencia S, Gendron P, Boucher G, Chagraoui J and Sauvageau G: $U B A P 2 L$ is amplified in a large subset of human lung adenocarcinoma and is critical for epithelial lung cell identity and tumor metastasis. FASEB J 31(11): 5012-5018, 2017. PMID: 28754713. DOI: 10.1096/fj.201601219RRR

$14 \mathrm{He}$ J, Chen Y, Cai L, Li Z and Guo X: Ubap2l silencing inhibits cell proliferation and $\mathrm{G}_{2} / \mathrm{M}$ phase transition in breast cancer. 
Breast Cancer 25(2): 224-232, 2018. PMID: 29196913. DOI 10.1007/s12282-017-0820-x

15 Crawford LJ, Walker B and Irvine AE: Proteasome inhibitors in cancer therapy. J Cell Commun Signal 5(2): 101-110, 2011. PMID: 21484190. DOI: 10.1007/s12079-011-0121-7

16 Iqbal M, Messina McLaughlin PA, Dunn D, Mallya S, Husten J, Ator MA and Chatterjee S: Proteasome inhibitors for cancer therapy. Bioorg Med Chem 20(7): 2362-2368, 2012. PMID: 22377673. DOI: 10.1016/j.bmc.2012.02.007

17 Rastogi N and Mishra DP: Therapeutic targeting of cancer cell cycle using proteasome inhibitors. Cell Div 7(1): 26, 2012. PMID: 23268747. DOI: 10.1186/1747-1028-7-26

18 Maeda M, Hasegawa H, Sugiyama M, Hyodo T, Ito S, Chen D, Asano E, Masuda A, Hasegawa Y, Hamaguchi M and Senga T: Arginine methylation of ubiquitin-associated protein 2-like is required for the accurate distribution of chromosomes. FASEB J 30(1): 312-323, 2016. PMID: 26381755. DOI: 10.1096/fj.14268987

19 Yang Y, Kitagaki J, Wang H, Hou DX and Perantoni AO: Targeting the ubiquitin-proteasome system for cancer therapy. Cancer Sci 100(1): 24-28, 2009. PMID: 19037995. DOI: 10.1111/j.1349-7006.2008.01013.x

20 Werness BA, Levine AJ and Howley PM: Association of human papillomavirus types 16 and 18 E6 proteins with p53. Science 248(4951): 76-79, 1990. PMID: 2157286. DOI: 10.1126/science. 2157286
21 Huang C, Chen Y, Dai H, Zhang H, Xie M, Zhang H, Chen F, Kang X, Bai X and Chen Z: UBAP2L arginine methylation by PRMT1 modulates stress granule assembly. Cell Death Differ, 2019. PMID: 31114027. DOI: 10.1038/s41418-019-0350-5

22 Anderson P and Kedersha N: Stress granules. Curr Biol 19(10): R397-398, 2009. PMID: 19467203. DOI: 10.1016/j.cub.2009. 03.013

23 Anderson P, Kedersha N and Ivanov P: Stress granules, p-bodies and cancer. Biochim Biophys Acta 1849(7): 861-870, 2015. PMID: 25482014. DOI: 10.1016/j.bbagrm.2014.11.009

24 Mahboubi $\mathrm{H}$ and Stochaj U: Cytoplasmic stress granules: Dynamic modulators of cell signaling and disease. Biochim Biophys Acta Mol Basis Dis 1863(4): 884-895, 2017. PMID: 28095315. DOI: 10.1016/j.bbadis.2016.12.022

25 Moeller BJ, Cao Y, Li CY and Dewhirst MW: Radiation activates HIF-1 To regulate vascular radiosensitivity in tumors: Role of reoxygenation, free radicals, and stress granules. Cancer Cell 5(5): 429-441, 2004. PMID: 15144951.

Received October 21, 2019

Revised November 3, 2019

Accepted November 5, 2019 